\title{
Spiroperoxy Lactones from Furans in One Pot: Synthesis of (+)- Premnalane A
}

loannis Margaros, Tamsyn Montagnon and Georgios Vassilikogiannakis*

Department of Chemistry, University of Crete, Vasilika Vouton, 71003 Iraklion, Crete, Greece

vasil@chemistry.uoc.gr 


\section{General Techniques:}

Reagents were purchased at the highest available commercial quality and used without further purification. All moisture sensitive reactions were carried out under an argon or a nitrogen atmosphere. Anhydrous solvents were obtained as follows: THF distilled from sodium and benzophenone; $\mathrm{CH}_{2} \mathrm{Cl}_{2}$ distilled from $\mathrm{CaH}_{2}$. Reactions were monitored by thin-layer chromatography (TLC) carried out on silica gel plates $\left(60 \mathrm{~F}_{254}\right)$ with UV light as the visualizing method and an acidic mixture of phosphomolybdic acid/cerium(IV) sulfate accompanied by heating of the plate as a developing system. The development agent contains $\mathrm{H}_{2} \mathrm{O}(94 \mathrm{~mL})$, concentrated $\mathrm{H}_{2} \mathrm{SO}_{4}(6 \mathrm{~mL}), \mathrm{Ce}\left(\mathrm{SO}_{4}\right)_{2} \cdot\left(\mathrm{H}_{2} \mathrm{O}\right) \mathrm{n}(1.0 \mathrm{~g})$ and phosphomolybdic acid (1.5 g). Column chromatography was carried out on $\mathrm{SiO}_{2}$ (silica gel 60, particle size $0.040-0.063 \mathrm{~mm}$ ) with the specified eluent.

${ }^{1} \mathrm{H}$ and ${ }^{13} \mathrm{C}$ NMR spectra were recorded respectively at $500 \mathrm{MHz}$ and $125 \mathrm{MHz}$ on a Bruker AMX 500 spectrometer, or at $300 \mathrm{MHz}$ and $75 \mathrm{MHz}$ on a Bruker MSL 300 spectrometer. All spectra are reported in $\delta(\mathrm{ppm})$ relative to the solvent residual peak (7.26 and $77.00 \mathrm{ppm}$ in $\mathrm{CDCl}_{3}$ ). The following abbreviations are used to explain the multiplicities: $\mathrm{s}=$ singlet, $\mathrm{d}=$ doublet, $\mathrm{t}=$ triplet, $\mathrm{q}=$ quartet, $\mathrm{m}=$ multiplet, $\mathrm{br}=$ broad.

Irradiation experiments (photoxygenations) were performed with a Xenon Variac Eimac Cermax $300 \mathrm{~W}$ lamp. 

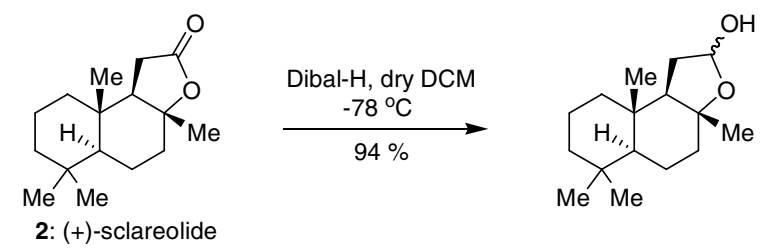

Diastereomeric lactols: To a solution of (+)-sclareolide (123 mg, $0.49 \mathrm{mmol}, 1.0$ equiv.) in dry $\mathrm{CH}_{2} \mathrm{Cl}_{2}(3.5 \mathrm{~mL})$, at $-78{ }^{\circ} \mathrm{C}$, was added dropwise Dibal-H (1.0 M in hexane, 593 $\mu \mathrm{L}, 0.59 \mathrm{mmol}, 1.2$ equiv.) over a period of $15 \mathrm{~min} .{ }^{[1]}$ The reaction was stirred for $30 \mathrm{~min}$ at $-78{ }^{\circ} \mathrm{C}$ and quenched at the same temperature by adding $\mathrm{H}_{2} \mathrm{O}(1 \mathrm{~mL})$. The mixture was allowed to warm to room temperature over $1 \mathrm{~h}$ and diluted with $\mathrm{CH}_{2} \mathrm{Cl}_{2}(10 \mathrm{~mL})$. An aqueous solution of Rochelle's salt was added $(9 \mathrm{~mL})$ and the mixture was vigorously stirred until organic phase became clear. The layers were separated and the organic phase dried over $\mathrm{MgSO}_{4}$ and then filtered. The filtrate was concentrated under reduced pressure to give the crude diastereomeric lactols (117 mg, 94\%, 1.0:0.7 ratio of diastereoisomers), which were used directly in the next step. The open hydroxyaldehyde form of these diastereomeric lactols was also detected in NMR spectra (8:1 lactol:hydroxyaldehyde).

Data for the hemiketal form of lactols: ${ }^{1} \mathrm{H}$ NMR $\left(300 \mathrm{MHz}, \mathrm{CDCl}_{3}\right): \delta=5.53-5.43(\mathrm{~m}$, $1 \mathrm{H}$ major $+1 \mathrm{H}$ minor), 4.51 (d, $J=3.3 \mathrm{~Hz}, 1 \mathrm{H}$, major), 4.39 (d, $J=4.2 \mathrm{~Hz}, 1 \mathrm{H}$, minor), $2.08-0.87(\mathrm{~m}, 14 \mathrm{H}$ major $+14 \mathrm{H}$ minor), 1.27 (s, 1 Me major), 1.07 (s, $1 \mathrm{Me}$ minor), 0.84 (br s, 1 Me major + 1 Me minor), 0.83 (s, 1 Me major), 0.79 (br s, 1 Me major + 2 Me minor) ppm; $\left.{ }^{13} \mathrm{C} \mathrm{NMR} \mathrm{(75} \mathrm{MHz,} \mathrm{CDCl}_{3}\right): \delta=98.8,97.1,83.1,81.5,60.7$ (2C), 57.0 (4C), 42.4 (2C), 40.2, 39.9, 39.7 (2C), 36.0 (2C), 33.4 (2C), 33.0 (2C), 31.5, 31.4, 21.0 (2C), 20.4 (2C), 18.3 (2C), 15.2 (2C) ppm; HRMS (ESI+): calcd for $\mathrm{C}_{16} \mathrm{H}_{28} \mathrm{O}_{2} \mathrm{Na}$ : $275.1987\left[\mathrm{M}+\mathrm{Na}^{+}\right]$, found: 275.1983 . 

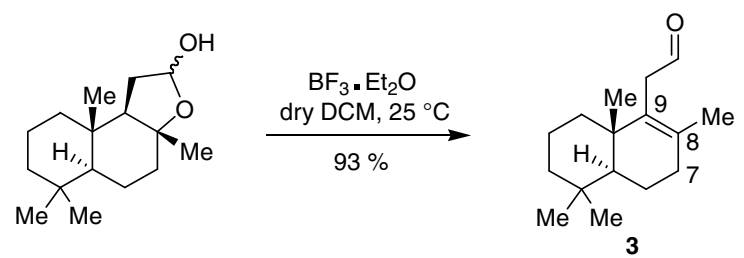

Enal 3: To a stirred solution of diastereomeric lactols $(117 \mathrm{mg}, 0.46 \mathrm{mmol})$ in dry $\mathrm{CH}_{2} \mathrm{Cl}_{2}(6 \mathrm{~mL})$, at $25^{\circ} \mathrm{C}$, was added $\mathrm{BF}_{3} \bullet \mathrm{Et}_{2} \mathrm{O}(70 \mu \mathrm{L}, 0.56 \mathrm{mmol}, 1.2$ equiv.) in a single portion (with as fast a rate as possible). The reaction mixture was stirred for a further 5 min before quenching with saturated aqueous $\mathrm{NaHCO}_{3}(1 \mathrm{~mL})$. Stirring was continued for $5 \mathrm{~min}$. The layers were separated and the organic phase dried over $\mathrm{MgSO}_{4}$ and then filtered. Crude enal 3, obtained after evaporation of the solvent under reduced pressure, required no further purification and was used directly in the next reaction (101 $\mathrm{mg}, 93 \%$, 3: $\Delta^{7,8}$ double bond regioisomer $=18: 1$, based on NMR).

Data for enal 3: ${ }^{1} \mathrm{H}$ NMR $\left(300 \mathrm{MHz}, \mathrm{CDCl}_{3}\right): \delta=9.50(\mathrm{t}, J=2.3 \mathrm{~Hz}, 1 \mathrm{H}), 3.09$ (br d, $J=$ $17.3 \mathrm{~Hz}, 1 \mathrm{H}$ ), 3.02 (br d, $J=17.3 \mathrm{~Hz}, 1 \mathrm{H}), 2.14-1.97$ (m, 2H), $1.74-1.33(\mathrm{~m}, 6 \mathrm{H}), 1.52$ (s, 3H), $1.24-0.92$ (m, 3H), 0.92 (s, 3H), 0.88 (s, 3H), 0.82 (s, 3H) ppm; ${ }^{13} \mathrm{C}$ NMR (75 $\left.\mathrm{MHz}, \mathrm{CDCl}_{3}\right): \delta=201.2,132.1,131.1,51.6,43.1,41.4,38.4,37.1,33.8,33.2,33.1,21.5$, 19.8, 19.7, 18.8 (2C) ppm; HRMS (ESI+): calcd for $\mathrm{C}_{16} \mathrm{H}_{26} \mathrm{ONa}$ : $257.1881\left[\mathrm{M}+\mathrm{Na}^{+}\right]$, found: 257.1880 .

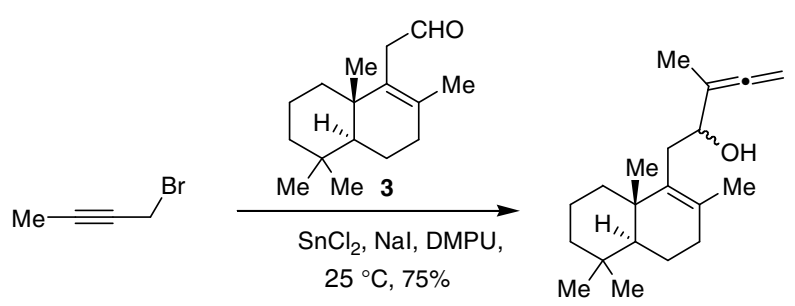

Diastereomeric allenic alcohols: To a solution of 1-bromo-2-butyne $(41 \mu \mathrm{L}, 0.45$ mmol, 1.05 equiv.), in dry DMPU ( $1.5 \mathrm{~mL}$ ), at $25{ }^{\circ} \mathrm{C}$, were added $\mathrm{SnCl}_{2}(95 \mathrm{mg}, 0.49$ mmol, 1.15 equiv.) and $\mathrm{NaI}(74 \mathrm{mg}, 0.49 \mathrm{mmol}, 1.15 \text { equiv. })^{[2]}$ The yellow colored reaction mixture was stirred, with light excluded from the reaction vessel, for $2 \mathrm{~h}$ wherein complete dissolution of solids was observed. To this reaction mixture, at $25^{\circ} \mathrm{C}$, a solution of enal 3 (101 mg, $0.43 \mathrm{mmol}, 1.0$ equiv.) in dry DMPU ( $0.7 \mathrm{~mL})$ was added dropwise. The orange reaction mixture was stirred in the dark for a further $20 \mathrm{~h}$. The reaction was 
diluted with $\mathrm{Et}_{2} \mathrm{O}(10 \mathrm{~mL})$ and quenched by addition of $30 \%$ aq. $\mathrm{NH}_{4} \mathrm{~F}(0.9 \mathrm{~mL})$. The layers were separated and the aqueous phase was extracted with $\mathrm{Et}_{2} \mathrm{O}(2 \times 5 \mathrm{~mL})$. The combined organic phases were then washed with $\mathrm{H}_{2} \mathrm{O}$, dried over $\mathrm{MgSO}_{4}$, filtered, and concentrated under reduced pressure. Purification by column chromatography (silica gel, hexanes/EtOAc $=30: 1 \rightarrow 20: 1)$ afforded each one of the diastereomeric alcohols separately (in a combined yield of $93 \mathrm{mg}, 75 \%, 1.6: 1$ ratio of less polar: more polar diastereomers).

Data for less polar diastereomer (major): ${ }^{1} \mathrm{H}$ NMR $\left(300 \mathrm{MHz}, \mathrm{CDCl}_{3}\right): \delta=4.75$ (dq, $J_{1}=$ $\left.3.2 \mathrm{~Hz}, J_{2}=2.1 \mathrm{~Hz}, 2 \mathrm{H}\right), 4.17$ (br d, $\left.J=8.9 \mathrm{~Hz}, 1 \mathrm{H}\right), 2.52-2.36(\mathrm{~m}, 1 \mathrm{H}), 2.28-1.94$ (m, $3 \mathrm{H}), 1.85(\mathrm{~d}, J=2.0 \mathrm{~Hz}, 1 \mathrm{H}), 1.77(\mathrm{t}, J=3.2 \mathrm{~Hz}, 3 \mathrm{H}), 1.66(\mathrm{~s}, 3 \mathrm{H}), 1.64-1.35(\mathrm{~m}, 5 \mathrm{H})$, $1.08-0.99$ (m, 3H), 0.97 (s, 3H), 0.89 (s, 3H), 0.84 (s, 3H) ppm; ${ }^{13} \mathrm{C}$ NMR (125 MHz, $\left.\mathrm{CDCl}_{3}\right): \delta=205.2,136.5,131.0,102.1,75.9,71.9,51.4,41.6,39.2,37.4,33.7,33.6$, 33.3, 33.2, 21.7, 20.9, 20.1, 19.0, 18.9, 14.5 ppm; HRMS (ESI+): calcd for $\mathrm{C}_{20} \mathrm{H}_{32} \mathrm{ONa}$ : $311.2351\left[\mathrm{M}+\mathrm{Na}^{+}\right]$, found: 311.2346 .

Data for more polar diastereomer (minor): ${ }^{1} \mathrm{H}$ NMR $\left(300 \mathrm{MHz}, \mathrm{CDCl}_{3}\right): \delta=4.75$ (dq, $J_{1}$ $\left.=3.2 \mathrm{~Hz}, J_{2}=2.1 \mathrm{~Hz}, 2 \mathrm{H}\right), 4.21(\mathrm{br} \mathrm{t}, J=6.0 \mathrm{~Hz}, 1 \mathrm{H}), 2.39(\mathrm{~d}, J=7.2 \mathrm{~Hz}, 2 \mathrm{H}), 2.08(\mathrm{dd}$, $\left.J_{1}=8.7 \mathrm{~Hz}, J_{2}=4.3 \mathrm{~Hz}, 2 \mathrm{H}\right), 1.76(\mathrm{t}, J=3.2 \mathrm{~Hz}, 3 \mathrm{H}), 1.63(\mathrm{~s}, 3 \mathrm{H}), 1.59-1.34(\mathrm{~m}, 6 \mathrm{H})$, $1.29-1.06(\mathrm{~m}, 3 \mathrm{H}), 1.02(\mathrm{~s}, 3 \mathrm{H}), 0.88(\mathrm{~s}, 3 \mathrm{H}), 0.85$ (s, 3H) ppm; ${ }^{13} \mathrm{C}$ NMR $(75 \mathrm{MHz}$, $\left.\mathrm{CDCl}_{3}\right): \delta=205.2,137.1,130.2,102.3,76.1,71.6,51.3,41.7,38.3,38.0,34.1,33.7$, 33.4, 33.3, 21.7, 21.4, 20.6, 19.1 (2C), 14.6 ppm; HRMS (ESI+): calcd for $\mathrm{C}_{20} \mathrm{H}_{32} \mathrm{ONa}$ : $311.2351\left[\mathrm{M}+\mathrm{Na}^{+}\right]$, found: 311.2346 . 

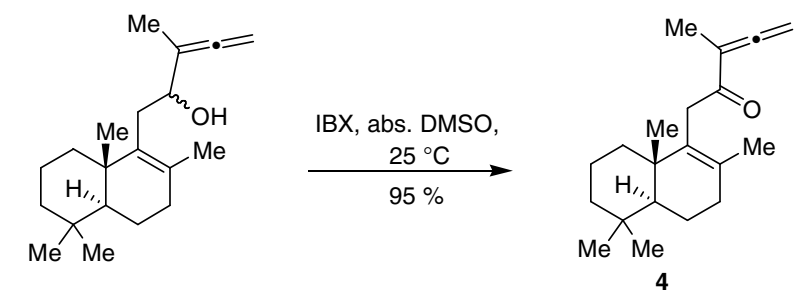

Allenic ketone 4: To a stirred solution of the diastereomeric allenic alcohols $(93 \mathrm{mg}, 0.32$ mmol, 1.0 equiv.), in dry DMSO (4 mL), at $25^{\circ} \mathrm{C}$, was added IBX (136 mg, $0.48 \mathrm{mmol}$, 1.5 equiv.). After 1.5 hours stirring the reaction was judged to be complete (based on TLC). The mixture was diluted with $\mathrm{Et}_{2} \mathrm{O}(15 \mathrm{~mL})$ and quenched by addition of saturated aq. $\mathrm{NaHCO}_{3}(4 \mathrm{~mL})$. The layers were separated and the organic phase washed with $\mathrm{H}_{2} \mathrm{O}$ ( $4 \times 4 \mathrm{~mL}$ ), dried over $\mathrm{MgSO}_{4}$, filtered and concentrated under reduced pressure to afford crude allenic ketone 4 (88 mg, $95 \%)$, which required no further purification and was directly used for the next reaction.

Data for allenic ketone 4: ${ }^{1} \mathrm{H}$ NMR $\left(300 \mathrm{MHz}, \mathrm{CDCl}_{3}\right): \delta=5.10(\mathrm{q}, J=3.0 \mathrm{~Hz}, 2 \mathrm{H}), 3.46$ $(\mathrm{d}, J=18.0 \mathrm{~Hz}, 1 \mathrm{H}), 3.27(\mathrm{~d}, J=18.0 \mathrm{~Hz}, 1 \mathrm{H}), 2.10\left(\mathrm{ddd}, J_{1}=17.8 \mathrm{~Hz}, J_{2}=10.8 \mathrm{~Hz}, J_{3}\right.$ $=7.4 \mathrm{~Hz}, 1 \mathrm{H}), 1.94\left(\mathrm{br} \mathrm{dd}, J_{1}=17.8 \mathrm{~Hz}, J_{2}=6.9 \mathrm{~Hz}, 1 \mathrm{H}\right), 1.73(\mathrm{t}, J=3.0 \mathrm{~Hz}, 3 \mathrm{H}), 1.67-$ 0.93(m, 9H), 1.40 (s, 3H), 0.84 (s, 3H), 0.83 (s, 3H), 0.77 (s, 3H) ppm; ${ }^{13} \mathrm{C}$ NMR (75 $\left.\mathrm{MHz}, \mathrm{CDCl}_{3}\right): \delta=215.4,198.9,134.6,129.3,103.1,78.3,51.1,41.4,38.2,37.7,36.1$, 33.3, 33.1, 33.0, 21.5, 20.0, 19.7, 18.9 (2C), 13.2 ppm; HRMS (ESI+): calcd for $\mathrm{C}_{20} \mathrm{H}_{30} \mathrm{ONa}$ : $309.2194\left[\mathrm{M}+\mathrm{Na}^{+}\right]$, found 309.2191.

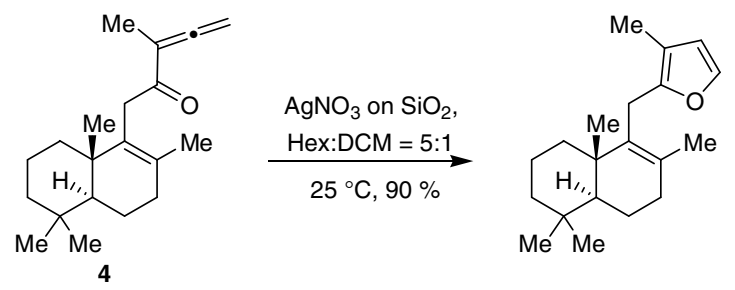

Furan: To a solution of allenic ketone 4 ( $88 \mathrm{mg}, 0.31 \mathrm{mmol}, 1.0$ equiv.), in hexane: $\mathrm{CH}_{2} \mathrm{Cl}_{2}(5: 1,3 \mathrm{~mL})$, at $25^{\circ} \mathrm{C}$, was added $\mathrm{AgNO}_{3}$ supported on $\mathrm{SiO}_{2}(10 \%$ w/w, $262 \mathrm{mgr}, 0.15 \mathrm{mmol}, 0.5$ equiv.). ${ }^{[3]}$ The suspension was stirred, with light carefully excluded from the reaction vessel, for $45 \mathrm{~min}$, before it was diluted with $\mathrm{Et}_{2} \mathrm{O}(5 \mathrm{~mL})$ and 
filtered through a short pad of cellite. After thorough washing of the cellite $\left(\mathrm{Et}_{2} \mathrm{O}\right)$, the solvent was removed in vacuo, to furnish crude furan as a pale yellow oil (79 $\mathrm{mg}, 90 \%)$. The crude material required no further purification and was used directly in the next reaction.

Data for furan: ${ }^{1} \mathrm{H}$ NMR $\left(500 \mathrm{MHz}, \mathrm{CDCl}_{3}\right): \delta=7.21(\mathrm{~d}, J=1.6 \mathrm{~Hz}, 1 \mathrm{H}), 6.12(\mathrm{~d}, J=1.6$ $\mathrm{Hz}, 1 \mathrm{H}), 3.32(\mathrm{~d}, J=16.1 \mathrm{~Hz}, 1 \mathrm{H}), 3.25(\mathrm{~d}, J=16.1 \mathrm{~Hz}, 1 \mathrm{H}), 2.13\left(\mathrm{ddd}, J_{1}=17.9 \mathrm{~Hz}, J_{2}\right.$ $\left.=10.9 \mathrm{~Hz}, J_{3}=7.4 \mathrm{~Hz}, 1 \mathrm{H}\right), 2.05\left(\mathrm{dd}, J_{1}=17.9 \mathrm{~Hz}, J_{2}=6.7 \mathrm{~Hz}, 1 \mathrm{H}\right), 1.96(\mathrm{~s}, 3 \mathrm{H}), 1.73-$ $1.65(\mathrm{~m}, 1 \mathrm{H}), 1.64(\mathrm{~s}, 3 \mathrm{H}), 1.63-1.35(\mathrm{~m}, 4 \mathrm{H}), 1.26(\mathrm{br} \mathrm{s}, 1 \mathrm{H}), 1.19\left(\mathrm{dd}, J_{1}=12.7 \mathrm{~Hz}, J_{2}\right.$ $=2.0 \mathrm{~Hz}, 1 \mathrm{H}), 1.12\left(\mathrm{dt}, J_{1}=13.3 \mathrm{~Hz}, J_{2}=3.9 \mathrm{~Hz}, 1 \mathrm{H}\right), 1.04\left(\mathrm{dt}, J_{1}=13.1 \mathrm{~Hz}, J_{2}=3.6\right.$ $\mathrm{Hz}, 1 \mathrm{H}), 0.93$ (s, 3H), 0.89 (s, 3H), 0.84 (s, 3H) ppm; $\left.{ }^{13} \mathrm{C} \mathrm{NMR} \mathrm{(125} \mathrm{MHz,} \mathrm{CDCl}_{3}\right): \delta=$ 150.3, 139.3, 136.3, 128.5, 112.8 (2C), 51.4, 41.6, 38.6, 36.5, 33.7, 33.3, 33.2, 24.6, 21.7, 20.1, 20.0, 19.0 (2C), 10.1 ppm; HRMS (ESI+): calcd for $\mathrm{C}_{20} \mathrm{H}_{30} \mathrm{ONa}$ : 309.2194 $\left[\mathrm{M}+\mathrm{Na}^{+}\right]$, found 309.2190 .
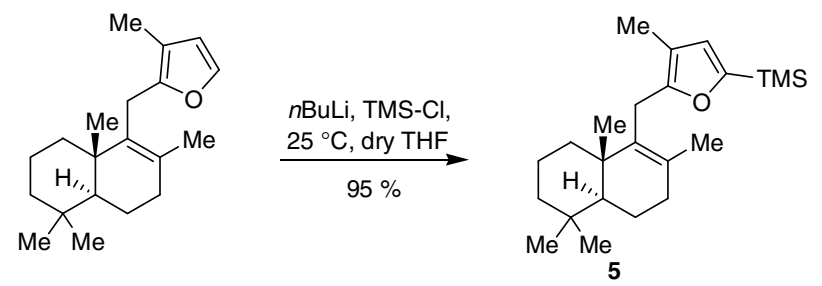

TMS-furan 5: To a stirred solution of furan ( $79 \mathrm{mg}, 0.28 \mathrm{mmol}, 1.0$ equiv.) in dry THF $(8 \mathrm{~mL})$ at $25^{\circ} \mathrm{C}$ was added dropwise $n$-BuLi (1.6 M in Hexane, $433 \mu \mathrm{L}, 0.69 \mathrm{mmol}, 2.5$ equiv.). After 10 min TMS-Cl ( $88 \mu \mathrm{L}, 0.69 \mathrm{mmol}, 2.5$ equiv.) was added at the same temperature, and the reaction mixture was stirred for a further $10 \mathrm{~min}$. The reaction mixture was diluted with $\mathrm{Et}_{2} \mathrm{O}(10 \mathrm{~mL})$ and quenched with $\mathrm{H}_{2} \mathrm{O}(2 \mathrm{~mL})$. The layers were separated and the organic phase dried over $\mathrm{MgSO}_{4}$, filtered, and then concentrated under reduced pressure to afford the silylated furan 5 as orange-yellow oil (94 mg, 95\%). Further purification of silylated furan $\mathbf{5}$ was not required and the material was used directly in the next reaction. 
Data for TMS-furan 5: ${ }^{1} \mathrm{H}$ NMR $\left(300 \mathrm{MHz}, \mathrm{CDCl}_{3}\right): \delta=6.37(\mathrm{~s}, 1 \mathrm{H}), 3.34(\mathrm{~d}, J=16.1$ $\mathrm{Hz}, 1 \mathrm{H}), 3.27$ (d, $J=16.1 \mathrm{~Hz}, 1 \mathrm{H}), 2.12-2.03(\mathrm{~m}, 2 \mathrm{H}), 1.94$ (s, 3H), $1.77-1.64$ (m, 2H), 1.62 (s, 3H), $1.59-1.33(\mathrm{~m}, 4 \mathrm{H}), 1.20-0.99(\mathrm{~m}, 3 \mathrm{H}), 0.90(\mathrm{~s}, 3 \mathrm{H}), 0.89$ (s, 3H), 0.84 (s, 3H), 0.20 (s, 9H) ppm; ${ }^{13} \mathrm{C}$ NMR $\left(75 \mathrm{MHz}, \mathrm{CDCl}_{3}\right): \delta=155.9,154.6,136.2$, $128.2,123.2,112.9,51.6,41.8,38.5,36.6,33.8,33.3,29.7,25.0,21.7,20.2,20.0,19.1$ (2C), 9.9, -1.5 (3C) ppm; HRMS (ESI+): calcd for $\mathrm{C}_{23} \mathrm{H}_{38} \mathrm{OSiNa}: 381.2590\left[\mathrm{M}+\mathrm{Na}^{+}\right]$, found 381.2587 .
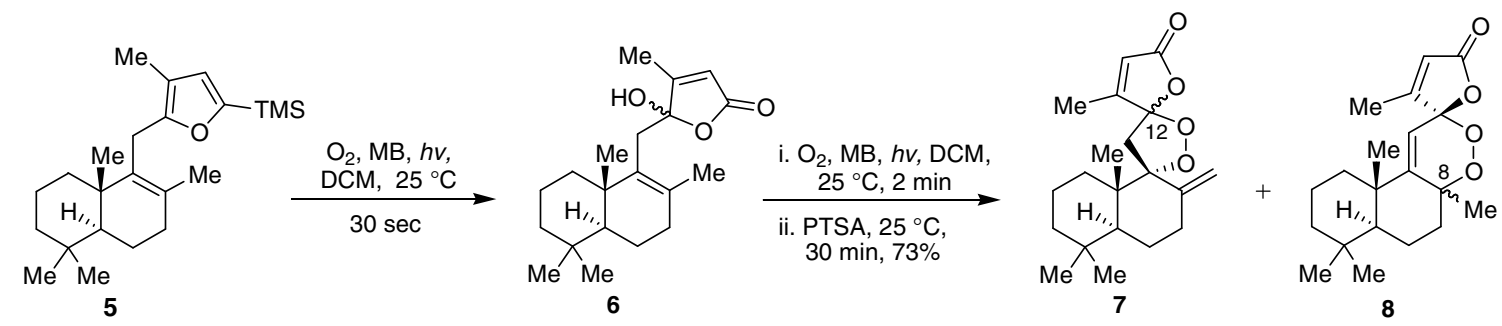

4-Hydroxy butenolide 6: A solution of silylated furan 5 (94 mg, $0.26 \mathrm{mmol}$ ) in $\mathrm{CHCl}_{3}$ $(10 \mathrm{~mL})$, containing Methylene Blue $\left(10^{-4} \mathrm{M}\right)$, at $8{ }^{\circ} \mathrm{C}$ (ice bath), was placed in a test tube with $\mathrm{O}_{2}$ gently bubbling through it. Irradiation with a Xenon Variac Eimac Cermax 300 W lamp for $30 \mathrm{sec}$ led to complete transformation of the starting material (based on TLC). The $\mathrm{CHCl}_{3}$ solution of diastereomeric 4-hydroxyl butenolides 6 may be used directly in the next step of the cascade sequence. For full characterization, however, the solvent may be removed in vacuo and the residue purified by flash column chromatography (silica gel, hexanes/EtOAc $=5: 1 \rightarrow 1: 1$ ) to afford 4-hydroxyl butenolides 6 as a 1.0:0.4 mixture of diastereomers.

Data for diastereomeric 4-hydroxyl butenolides 6: ${ }^{1} \mathrm{H}$ NMR (500 MHz, $\mathrm{CDCl}_{3}$ ): $\delta=5.82$ (d, $J=1.2 \mathrm{~Hz}, 1 \mathrm{H}$, major), 5.80 (d, $J=1.3 \mathrm{~Hz}, 1 \mathrm{H}$, minor), 3.92 (br s, OH, major), 3.89 (br s, OH, minor), 2.85 (d, $J=15.2 \mathrm{~Hz}, 1 \mathrm{H}$, minor), 2.82 (d, $J=15.2 \mathrm{~Hz}, 1 \mathrm{H}$, major), 2.56 (d, $J=15.2 \mathrm{~Hz}, 1 \mathrm{H}$, major), 2.45 (d, $J=15.2 \mathrm{~Hz}, 1 \mathrm{H}$, minor), $2.31-2.14$ (m, $2 \mathrm{H}$ major + 2H minor), 2.13 (d, $J=1.3 \mathrm{~Hz}, 3 \mathrm{H}$, minor), 2.11 (d, $J=1.2 \mathrm{~Hz}, 3 \mathrm{H}$, major), 2.06 (br s, 1H minor), 2.04 (br s, 1H major), 1.95 (br d, $J=12.0 \mathrm{~Hz}, 1 \mathrm{H}$ major $+1 \mathrm{H}$ minor), $1.87-1.70(\mathrm{~m}, 1 \mathrm{H}$ major $+1 \mathrm{H}$ minor), 1.69 (s, 3H, minor), 1.68 (s, 3H, major), $1.65-$ 
$1.19(\mathrm{~m}, 4 \mathrm{H}$ major $+4 \mathrm{H}$ minor), 1.17 (s, 3H, major), $1.15-1.00(\mathrm{~m}, 2 \mathrm{H}$ major $+2 \mathrm{H}$ minor), 0.95 (s, 3H, minor), 0.90 (s, 3H, minor), 0.89 (s, 3H, major), 0.87 (s, 3H, major), 0.84 (s, 3H, minor) ppm; ${ }^{13} \mathrm{C}$ NMR $\left(75 \mathrm{MHz}, \mathrm{CDCl}_{3}\right): \delta=170.2(2 \mathrm{C}), 167.7,167.4$, 136.4, 136.0, 134.5, 134.1, 118.6, 118.4, 105.4, 105.3, 51.3 (2C), 41.6, 41.5, 38.7, 37.8, 37.7 (2C), 34.6 (2C), 34.0, 33.9, 33.5, 33.4 (2C), 33.3, 29.7 (2C), 21.7, 21.1, 20.8, 20.5, 19.1, 18.9, 18.8 (2C), 12.9, 12.7 ppm; HRMS (ESI+): calcd for $\mathrm{C}_{20} \mathrm{H}_{30} \mathrm{O}_{3} \mathrm{Na}: 341.2093$ $\left[\mathrm{M}+\mathrm{Na}^{+}\right]$, found: 341.2088 .

(+)-Premnalane A (8b), 8-epi-Premnalene A (8a) and [5,5]-endoperoxy spirolactones (7a/b): A test tube containing a solution of 4-hydroxy butenolides 6 (77 $\mathrm{mg}, 0.24 \mathrm{mmol})$ in $\mathrm{CHCl}_{3}(10 \mathrm{~mL})$ and Methylene Blue $\left(10^{-4} \mathrm{M}\right)$, at $8{ }^{\circ} \mathrm{C}$ (ice bath), was irradiated with a Xenon Variac Eimac Cermax $300 \mathrm{~W}$ lamp, while $\mathrm{O}_{2}$ was gently bubbled through the solution. After $2.5 \mathrm{~min}$ of irradiation complete consumption of the starting material was observed by TLC. Catalytic PTSA was added and the reaction mixture was stirred for 30 $\min$ at $25{ }^{\circ} \mathrm{C}$. NMR spectra of the crude reaction mixture revealed the presence of $[5,5]$ endoperoxy spirolactones $\mathbf{7 a} / \mathbf{b}$ (10:1 mixture of diastereomers $\mathbf{7 a : 7 b})$ and [6,5]endoperoxy spirolactones $\mathbf{8 a / b}(1: 1$ mixture of diastereomers $\mathbf{8 a : 8 b})$ in a 2:1 ratio ([5,5]endoperoxy spirolactones:[6,5]-endoperoxy spirolactones, see Table 1). The solvent was removed in vacuo and the reaction products were separated by flash column chromatography (silica gel, hexanes/EtOAc $=15: 1 \rightarrow 2: 1$ ). The combined isolated yield was $54 \mathrm{mg}$ for all these products (73\% combined yield, over three steps). The only products which could not readily be separated by flash column chromatography were (+)premnalane A (8b) and its 8-epi-isomer (8a). Selective crystallization of (+)-premnalane A from its epimer was achieved in $\mathrm{MeOH}$. Despite the fact that the two diastereoisomeres are inseparable by flash chromatography we managed to collect a few fractions which contain a small amount of very pure (+)-premnalane A $(\mathbf{8 b})$ and measure its optical rotation: $[\alpha]_{D}^{24}+120\left(c 0.5, \mathrm{CHCl}_{3}\right)$.

Data for [6,5]-endoperoxy spirolactones 8a/b: ${ }^{1} \mathrm{H}$ NMR (500 MHz, $\left.\mathrm{CDCl}_{3}\right): \delta=5.96(\mathrm{~m}$, $1 \mathrm{H}+1 \mathrm{H}), 5.23(\mathrm{~s}, 1 \mathrm{H}), 5.18(\mathrm{~s}, 1 \mathrm{H}), 1.98(\mathrm{~d}, J=1.5 \mathrm{~Hz}, 3 \mathrm{H}), 1.95(\mathrm{~d}, J=1.6 \mathrm{~Hz}, 3 \mathrm{H})$, $1.94-1.69(\mathrm{~m}, 6 \mathrm{H}), 1.68(\mathrm{~s}, 3 \mathrm{H}), 1.66(\mathrm{~s}, 3 \mathrm{H}), 1.66-1.23(\mathrm{~m}, 10 \mathrm{H}), 1.21(\mathrm{~s}, 3 \mathrm{H}), 1.20-$ 
$1.13(\mathrm{~m}, 4 \mathrm{H}), 1.12(\mathrm{~s}, 3 \mathrm{H}), 1.09-1.04(\mathrm{~m}, 2 \mathrm{H}), 0.93(\mathrm{~s}, 3 \mathrm{H}), 0.90(\mathrm{~s}, 3 \mathrm{H}), 0.89(\mathrm{~s}, 3 \mathrm{H})$, 0.88 (s, 3H) ppm; ${ }^{13} \mathrm{C}$ NMR (125 MHz, $\left.\mathrm{CDCl}_{3}\right): \delta=169.5$ (2C), 163.1, $162.4(2 \mathrm{C})$, 160.4, 121.1 (2C), 111.2, 109.4, 107.1, 106.9, 79.7, 78.9, 43.6, 41.8, 41.5, 40.3, 38.8, $38.4,37.3,36.1,33.8,33.5,33.0,32.9,29.6,26.3,25.8,25.6,25.3,21.6,21.5,21.2,19.0$, 18.7, 18.5, 16.5, 13.0, 12.9 ppm; HRMS (ESI+): calcd for $\mathrm{C}_{20} \mathrm{H}_{28} \mathrm{O}_{4} \mathrm{Na}$ : 355.1885 $\left[\mathrm{M}+\mathrm{Na}^{+}\right]$, found: 355.1881 .

Data for the major [5,5]-endoperoxy spirolactone 7a: ${ }^{1} \mathrm{H} \mathrm{NMR}\left(300 \mathrm{MHz}, \mathrm{CDCl}_{3}\right): \delta=$ $5.94(\mathrm{q}, J=1.5 \mathrm{~Hz}, 1 \mathrm{H}), 5.13(\mathrm{~d}, J=1.5 \mathrm{~Hz}, 1 \mathrm{H}), 4.88(\mathrm{~s}, 1 \mathrm{H}), 3.10(\mathrm{~d}, J=14.3 \mathrm{~Hz}, 1 \mathrm{H})$, $2.85(\mathrm{~d}, J=14.3 \mathrm{~Hz}, 1 \mathrm{H}), 2.67$ (br dt, $J_{1}=13.1, J_{2}=5.4, \mathrm{~Hz}, 1 \mathrm{H}$ ), 2.34 (ddd, $J_{1}=12.9$, $\left.J_{2}=4.1, J_{3}=2.3 \mathrm{~Hz}, 1 \mathrm{H}\right), 2.05(\mathrm{~d}, J=1.5 \mathrm{~Hz}, 3 \mathrm{H}), 2.02-1.94(\mathrm{~m}, 1 \mathrm{H}), 1.83-1.73(\mathrm{~m}$, $1 \mathrm{H}), 1.65-1.20(\mathrm{~m}, 7 \mathrm{H}), 0.88$ (s, 3H), 0.84 (s, 3H), 0.80 (s, 3H) ppm; ${ }^{13} \mathrm{C}$ NMR $(75$ $\left.\mathrm{MHz}, \mathrm{CDCl}_{3}\right): \delta=169.1,159.8,148.0,121.7,113.9,110.4,94.8,48.0,42.8,41.4,41.2$, 33.9, 33.2, 32.0, 29.7, 23.6, 21.9, 18.7, 18.4, 13.1 ppm; HRMS (ESI+): calcd for $\mathrm{C}_{20} \mathrm{H}_{28} \mathrm{O}_{4} \mathrm{Na}: 355.1885\left[\mathrm{M}+\mathrm{Na}^{+}\right]$, found: 355.1881 .

[5,5]-endoperoxy spirolactone 7b: A solution of 6 (30 mg, $0.082 \mathrm{mmol})$ in $\mathrm{MeOH}: \mathrm{DCM}$ (6:1, $3 \mathrm{~mL})$ and Methylene Blue $\left(10^{-4} \mathrm{M}\right)$, at $8{ }^{\circ} \mathrm{C}$ (ice bath), was irradiated with a Xenon Variac Eimac Cermax $300 \mathrm{~W}$ lamp, for 3.5 min, whilst $\mathrm{O}_{2}$ was gently bubbled through the solution. Catalytic PTSA was added and the reaction mixture was stirred for $30 \mathrm{~min}$ at $25{ }^{\circ} \mathrm{C}$. According to reaction's crude NMR spectrum, diastereomeric [5,5]-endoperoxy spirolactones $7(\mathbf{7 a : 7 b}=1: 3)$ and diastereomeric [6,5]-endoperoxy spirolactones $8(\mathbf{8 a : 8 b}$ $=1: 1$ ) were prepared in a 4:1 ratio (see Table 1). The solvent was removed in vacuo and the products were separated by flash column chromatography (silica gel, hexanes/EtOAc $=15: 1 \rightarrow 2: 1)$. The combined isolated yield was $19 \mathrm{mg}$ for all these products $(70 \%$ combined yield, over three steps).

Data for the major [5,5]-endoperoxy spirolactone $7 \mathbf{b}:{ }^{1} \mathrm{H}$ NMR $\left(500 \mathrm{MHz}, \mathrm{CDCl}_{3}\right): \delta=$ $5.93(\mathrm{q}, J=1.1 \mathrm{~Hz}, 1 \mathrm{H}), 5.09(\mathrm{~d}, J=0.7 \mathrm{~Hz}, 1 \mathrm{H}), 5.01(\mathrm{~s}, 1 \mathrm{H}), 2.99(\mathrm{~d}, J=13.9 \mathrm{~Hz}, 1 \mathrm{H})$, $2.94(\mathrm{~d}, J=13.9 \mathrm{~Hz}, 1 \mathrm{H}), 2.43\left(\mathrm{br} \mathrm{dt}, J_{1}=13.2, J_{2}=4.5, \mathrm{~Hz}, 1 \mathrm{H}\right), 2.39-2.33(\mathrm{~m}, 1 \mathrm{H}), 2.13$ $(\mathrm{d}, J=1.1 \mathrm{~Hz}, 3 \mathrm{H}), 1.83\left(\mathrm{dt}, J_{1}=12.0, J_{2}=4.9,1 \mathrm{H}\right), 1.76-1.70(\mathrm{~m}, 1 \mathrm{H}), 1.68-1.48(\mathrm{~m}$, 
$3 \mathrm{H}), 1.45-1.23(\mathrm{~m}, 4 \mathrm{H}), 0.91(\mathrm{~s}, 3 \mathrm{H}), 0.84(\mathrm{~s}, 3 \mathrm{H}), 0.82(\mathrm{~s}, 3 \mathrm{H}) \mathrm{ppm} ;{ }^{13} \mathrm{C}$ NMR (125 $\left.\mathrm{MHz}, \mathrm{CDCl}_{3}\right): \delta=168.6,160.5,144.3,121.0,113.4,111.3,94.1,48.2$, 43.5, 41.3, 41.0, 33.8, 33.2, 32.0, 29.7, 23.0, 21.8, 18.9, 17.3, 12.7 ppm; HRMS (ESI+): calcd for $\mathrm{C}_{20} \mathrm{H}_{28} \mathrm{O}_{4} \mathrm{Na}: 355.1885$ [M+Na ${ }^{+}$, found: 355.1881 .

[1] L. Ferrie, S. Reymond, P. Capdevielle, J. Cossy, Org. Lett. 2006, 8, 3441.

[2] J. D. Winkler, K. J. Quinn, C. H. MacKinnon, S. D. Hiscock, E. C. McLaughlin, Org. Lett. 2003, 5, $1805-1808$.

[3] J. A. Marshall, C. A. Sehon, J. Org. Chem. 1995, 60, 5966. 

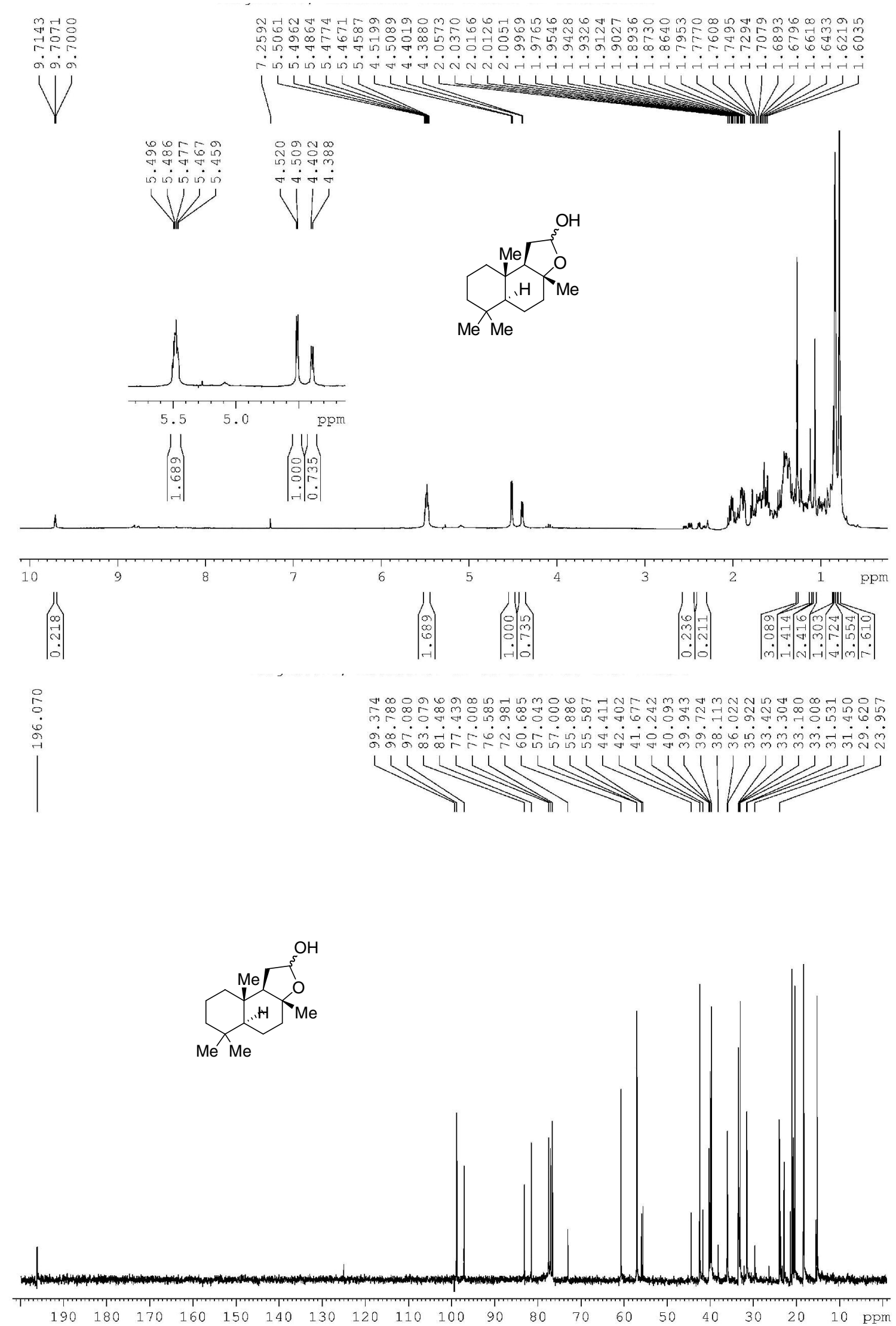


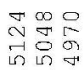

$$
\begin{aligned}
& \text { नंबं }
\end{aligned}
$$

V
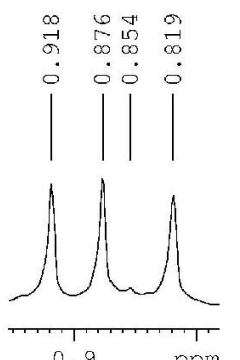

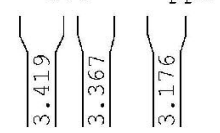
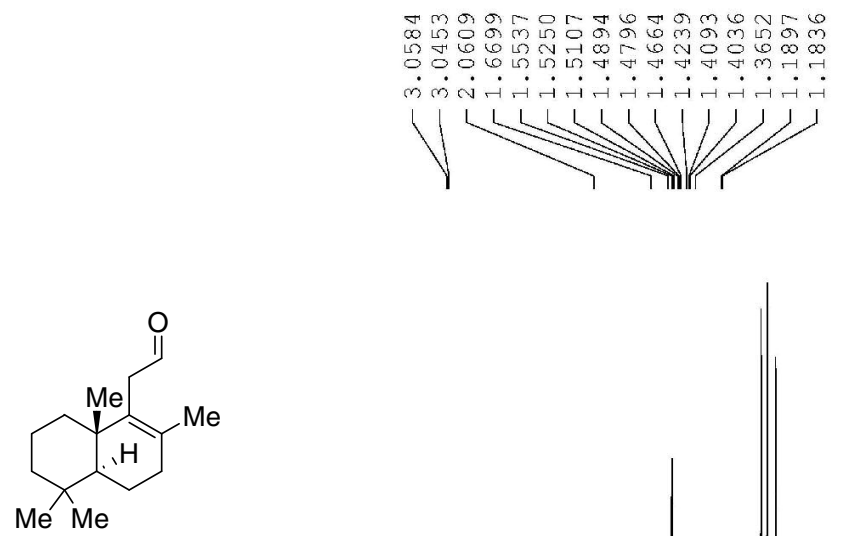

$\mathrm{Me} \mathrm{Me}$
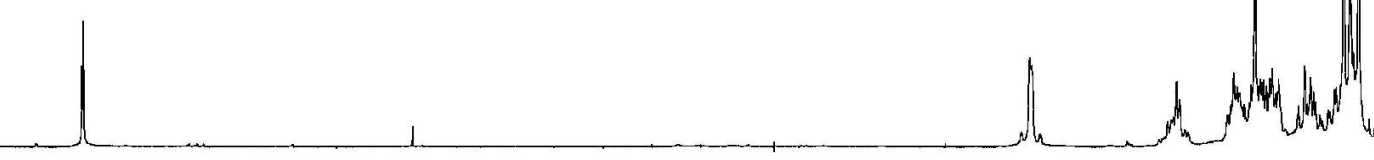

$$
\begin{aligned}
& 7 \\
& 10
\end{aligned}
$$

7
10

$\left|\begin{array}{c}10 \\ 0 \\ 0 \\ -1\end{array}\right|$

9

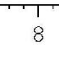

7

6

5
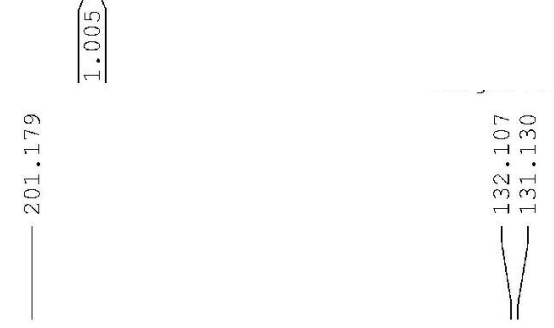

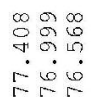

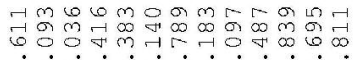

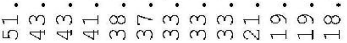

$1 /$
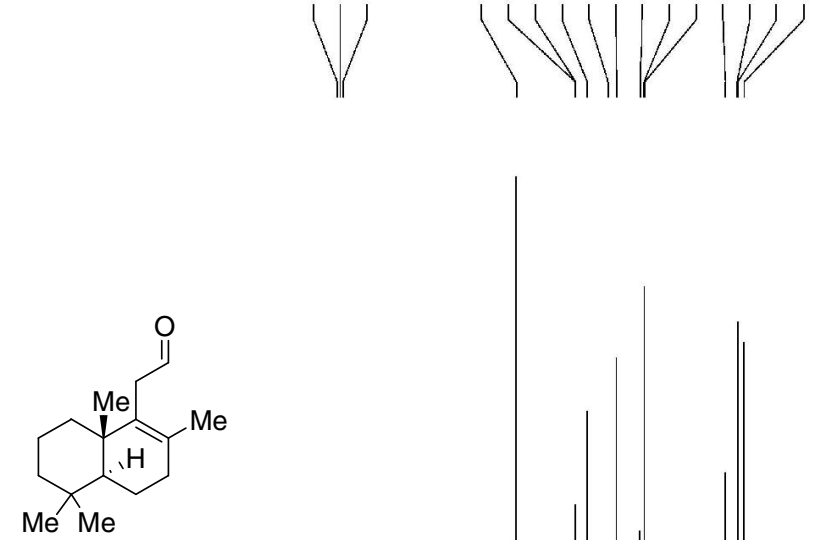

$\mid$
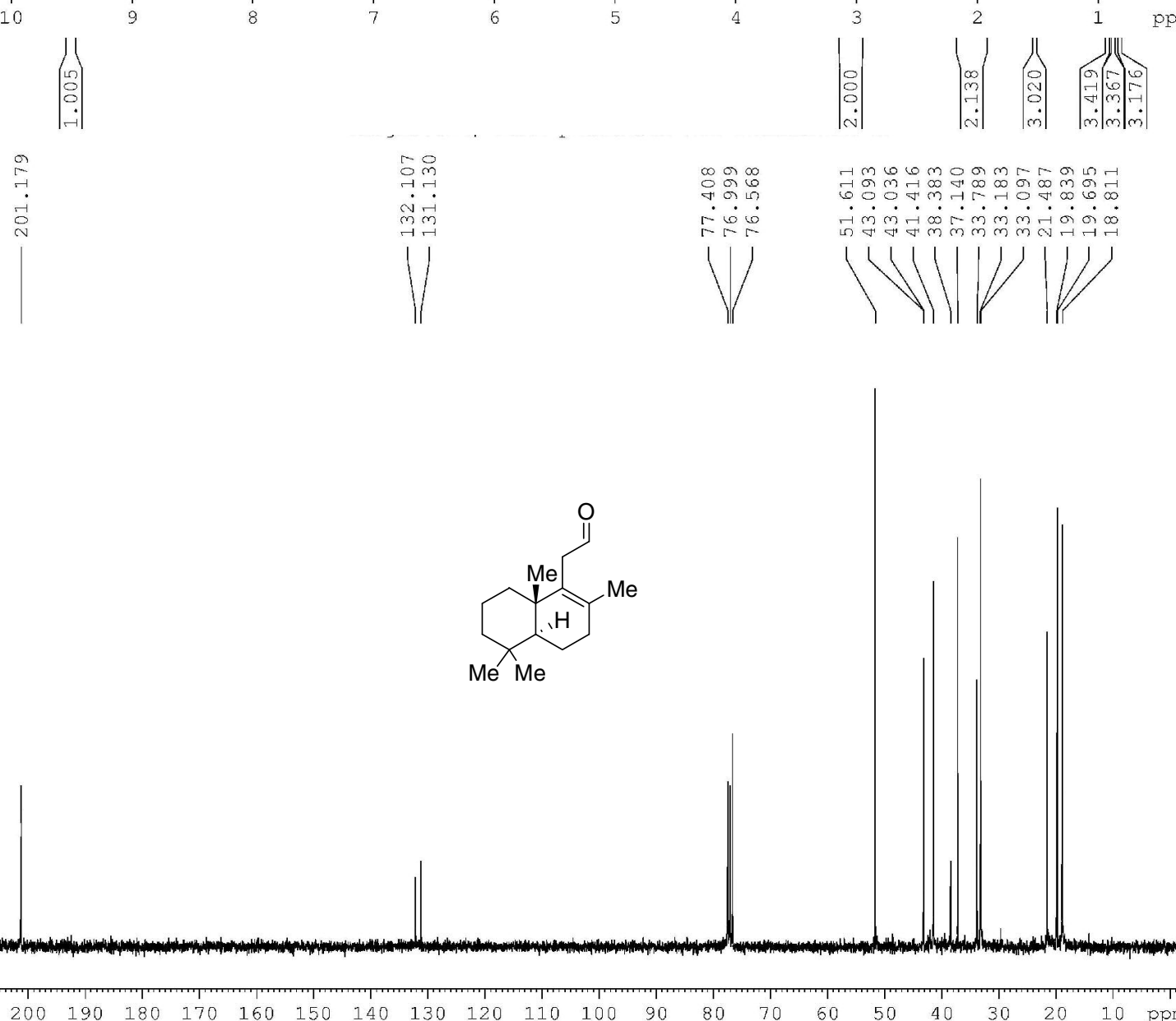

$\begin{array}{llllll}200 & 190 & 180 & 170 & 160 & 150\end{array}$
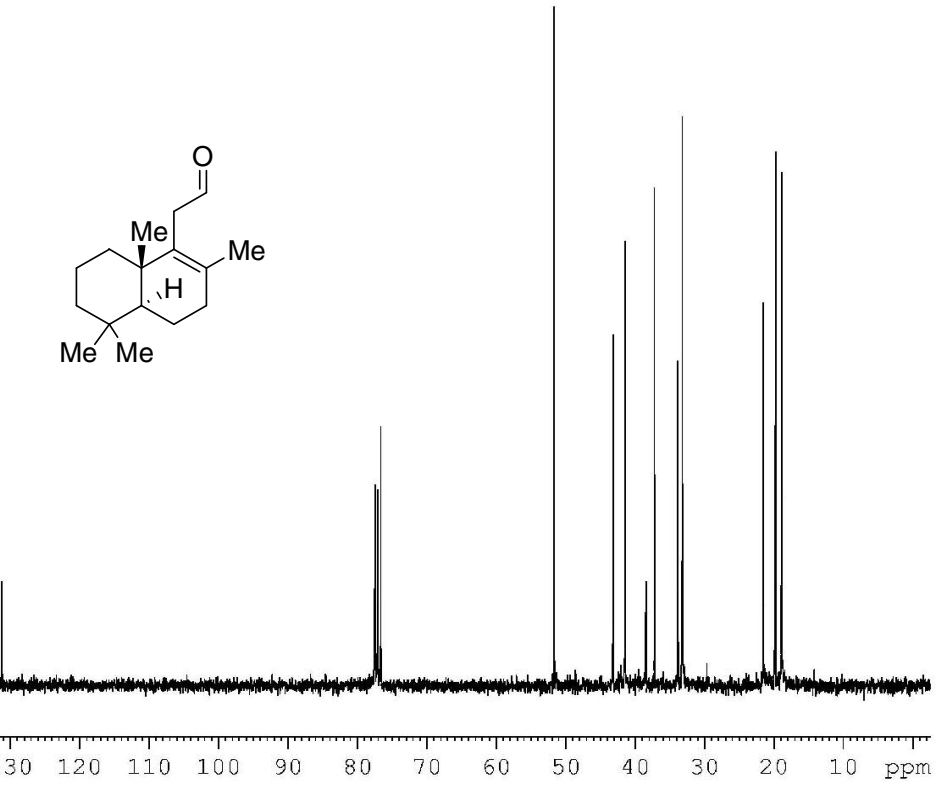

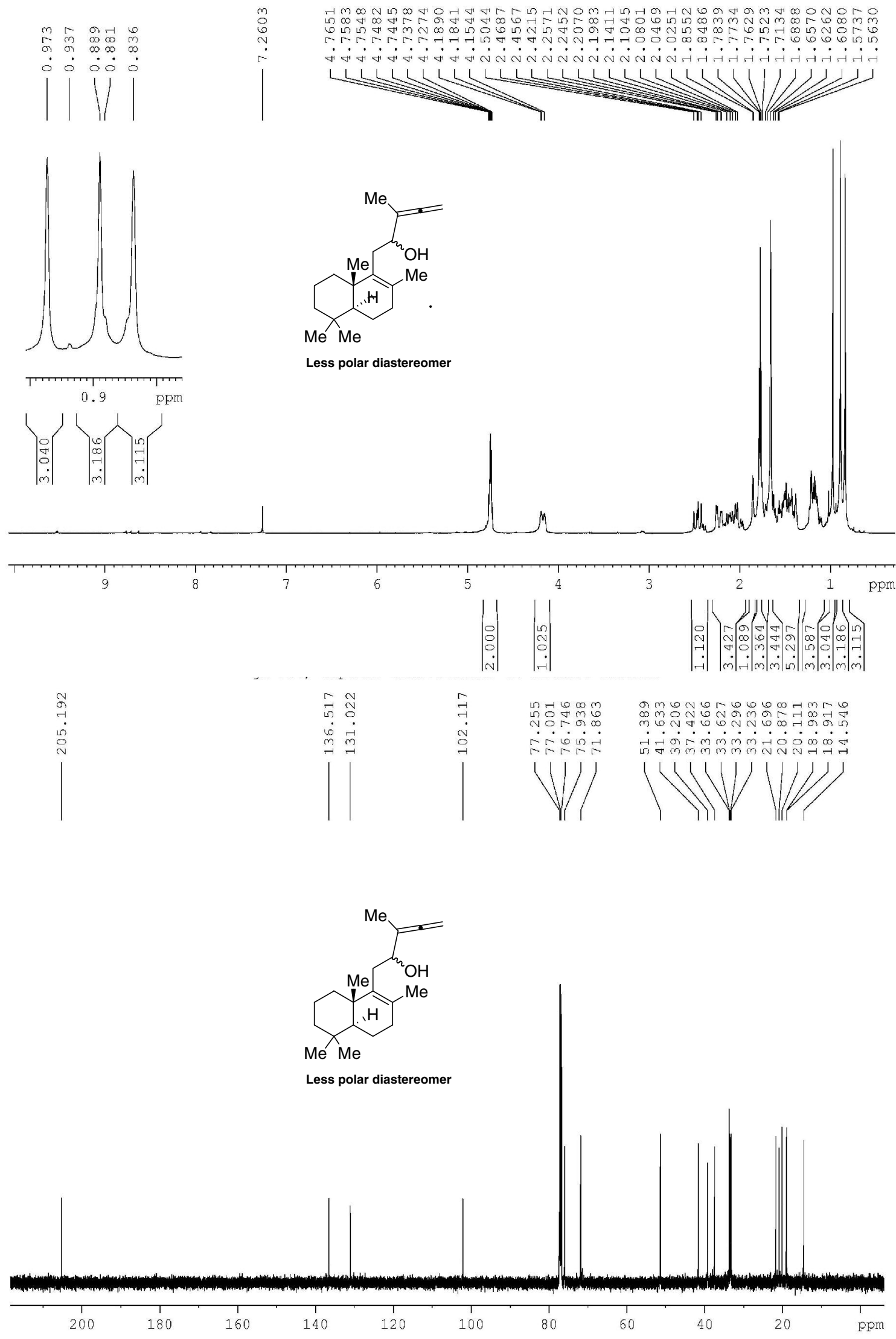


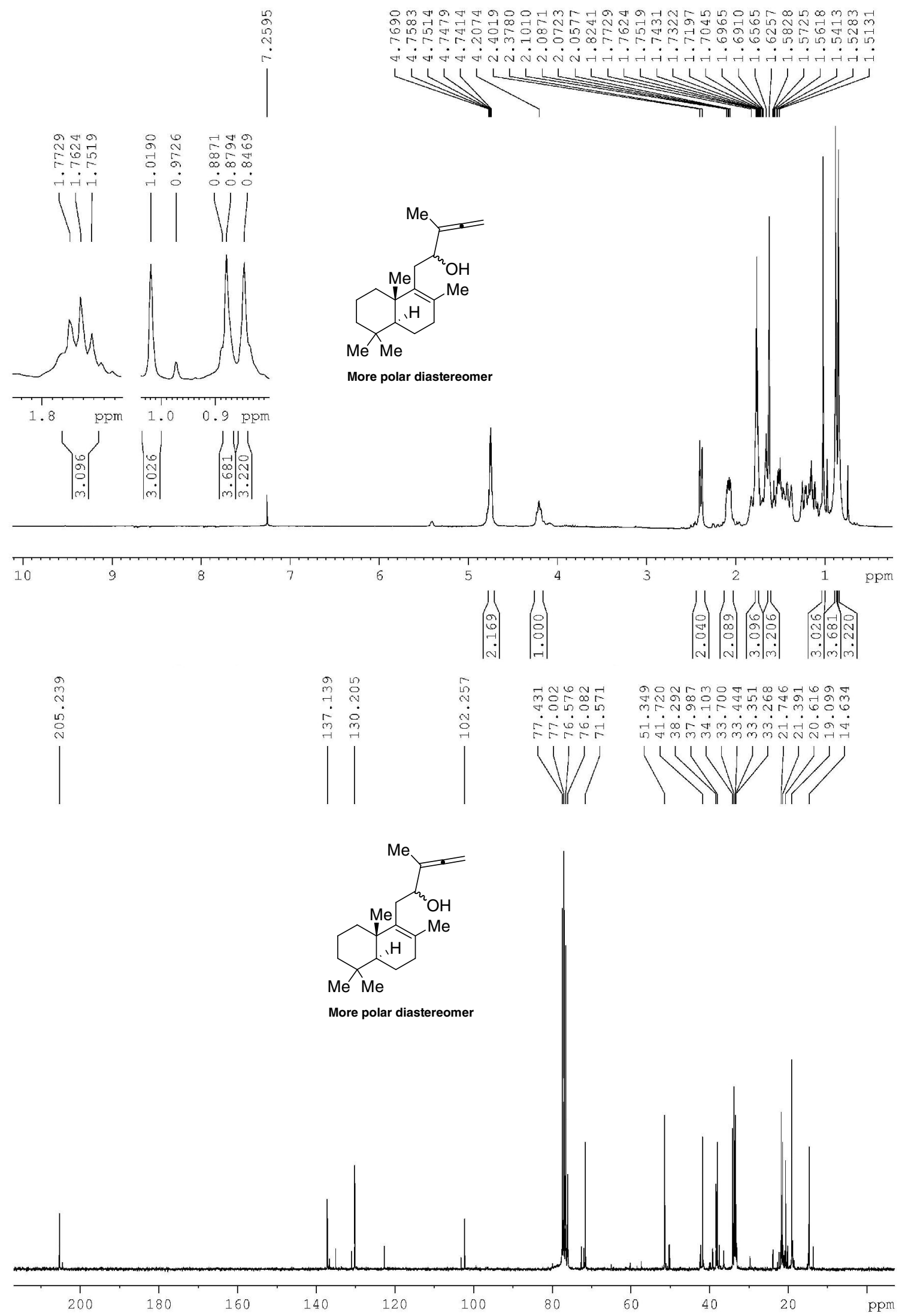



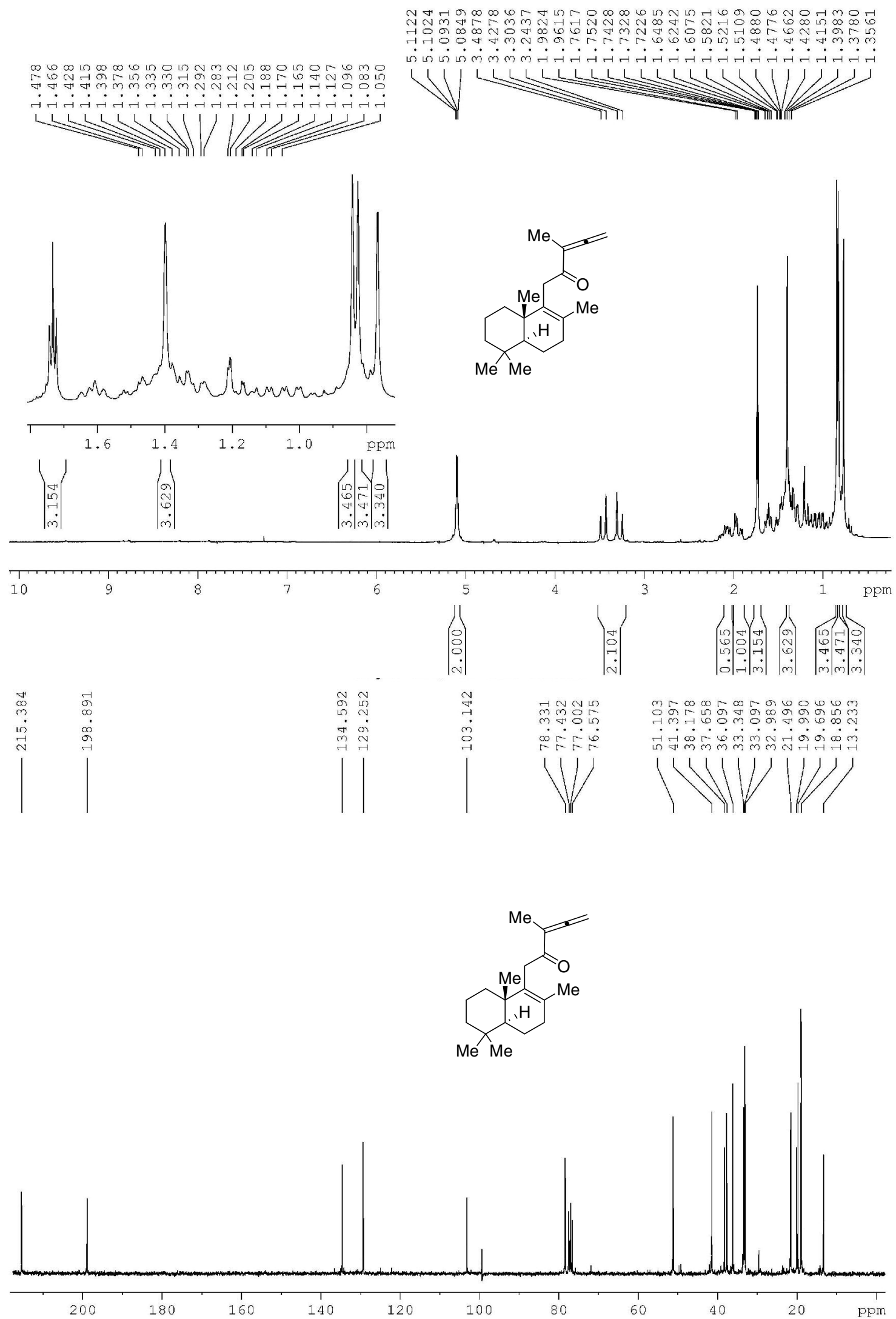

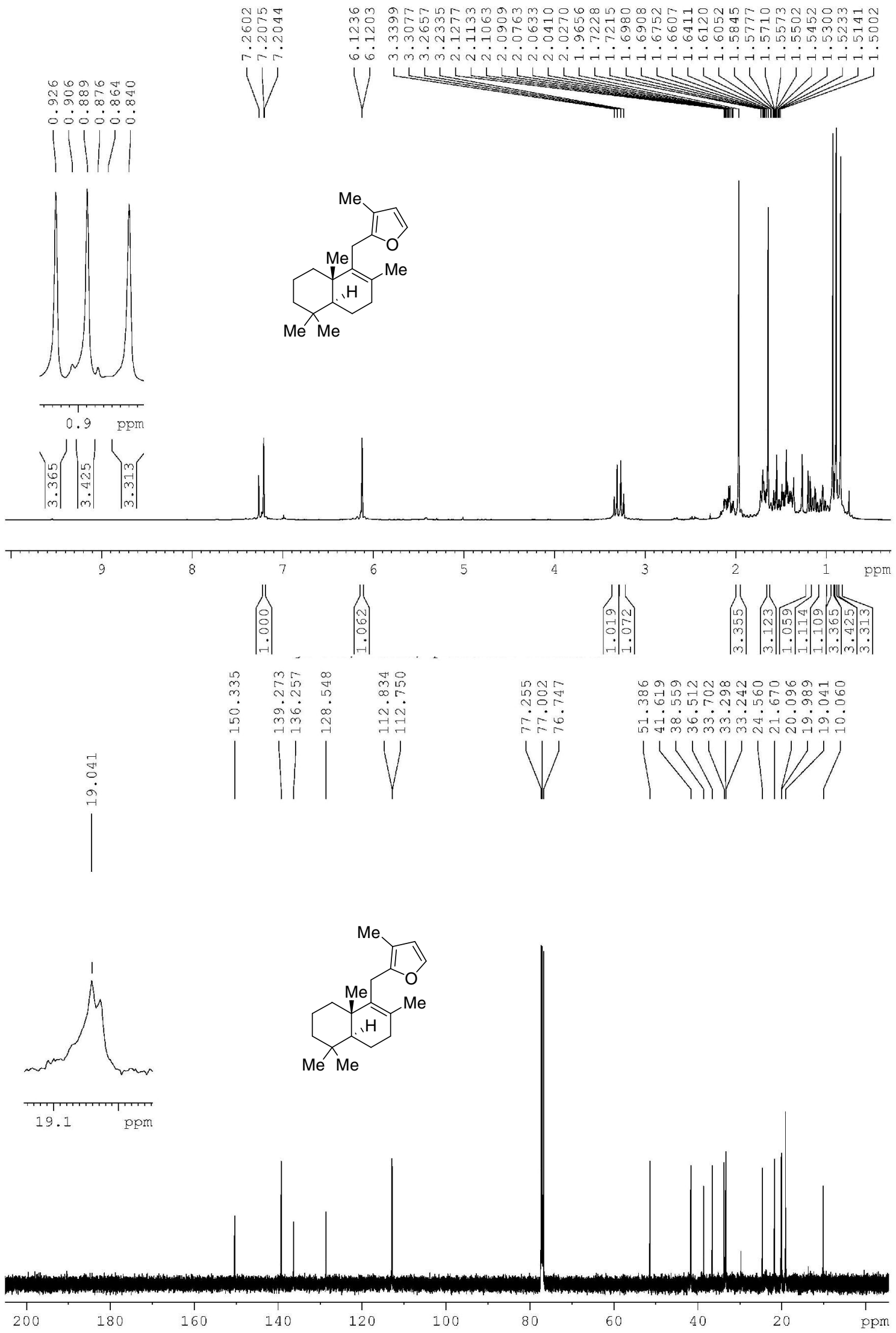

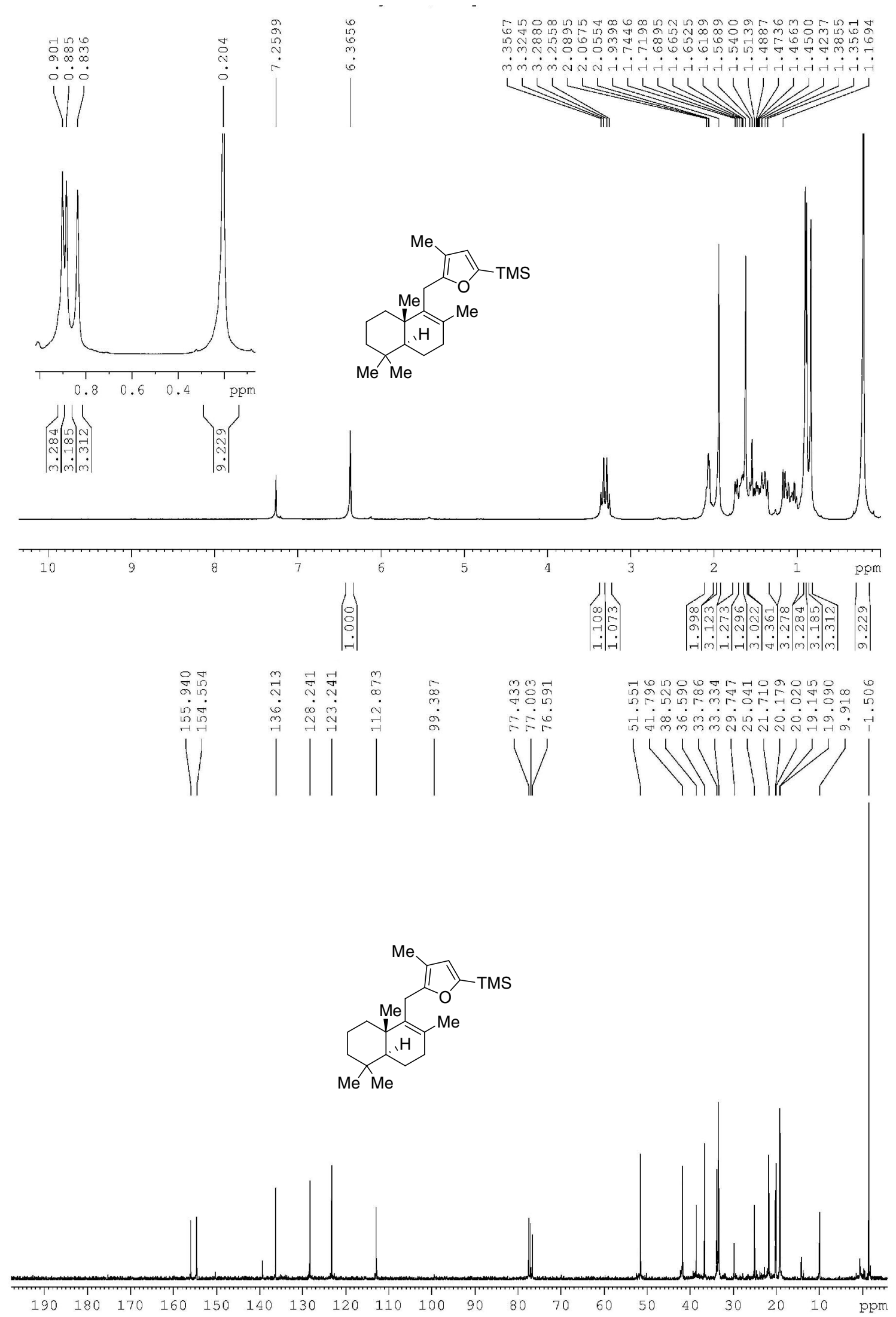


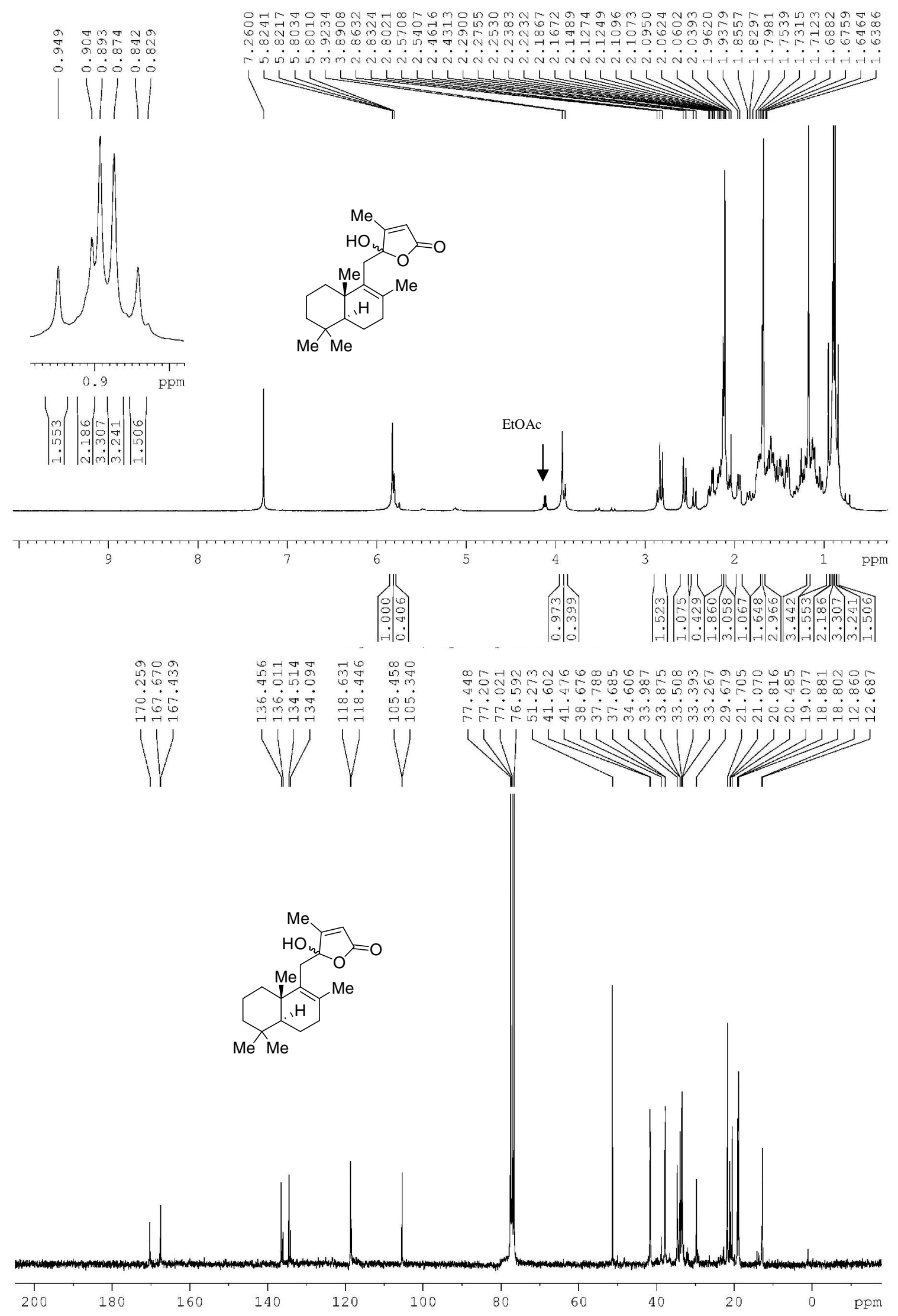



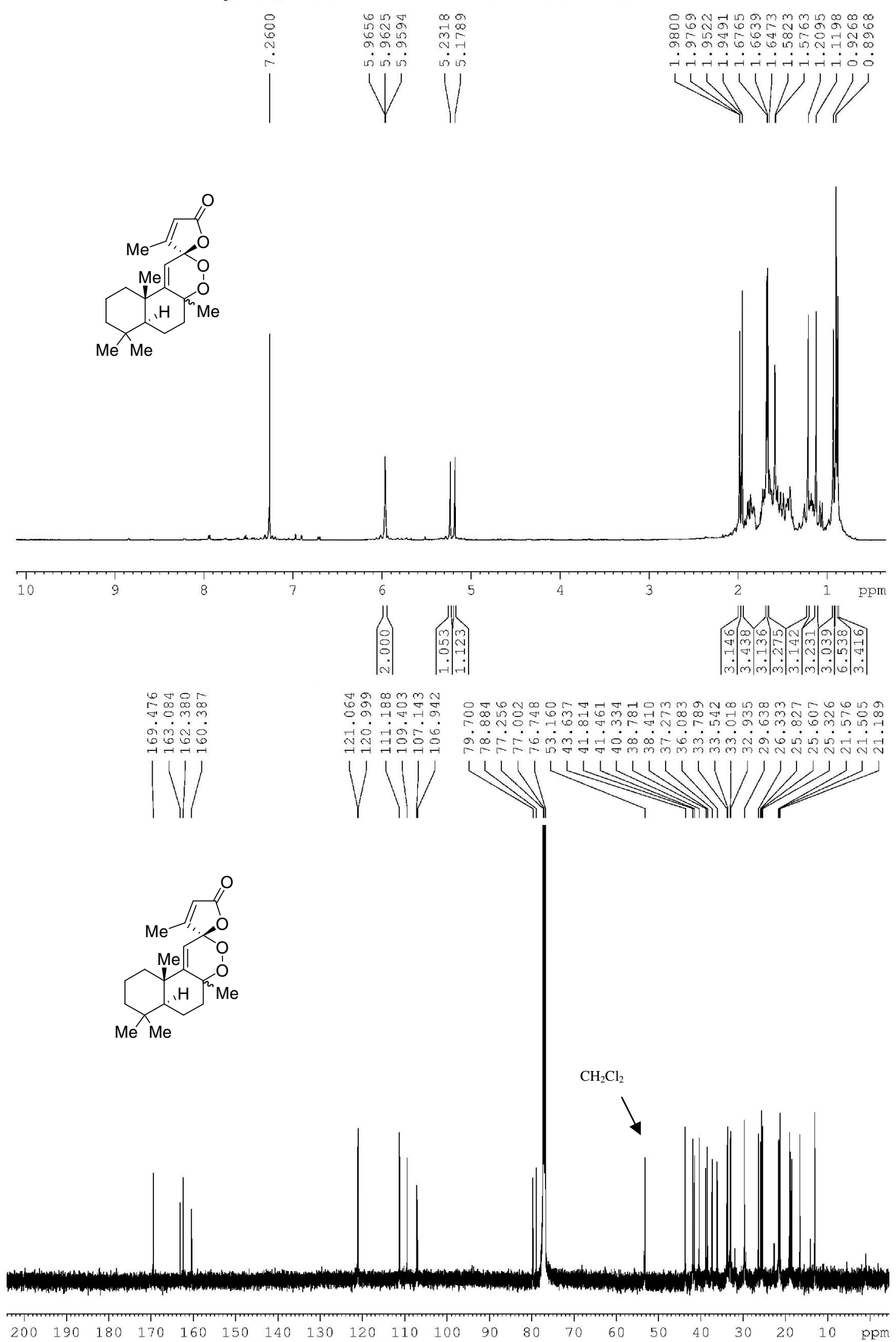


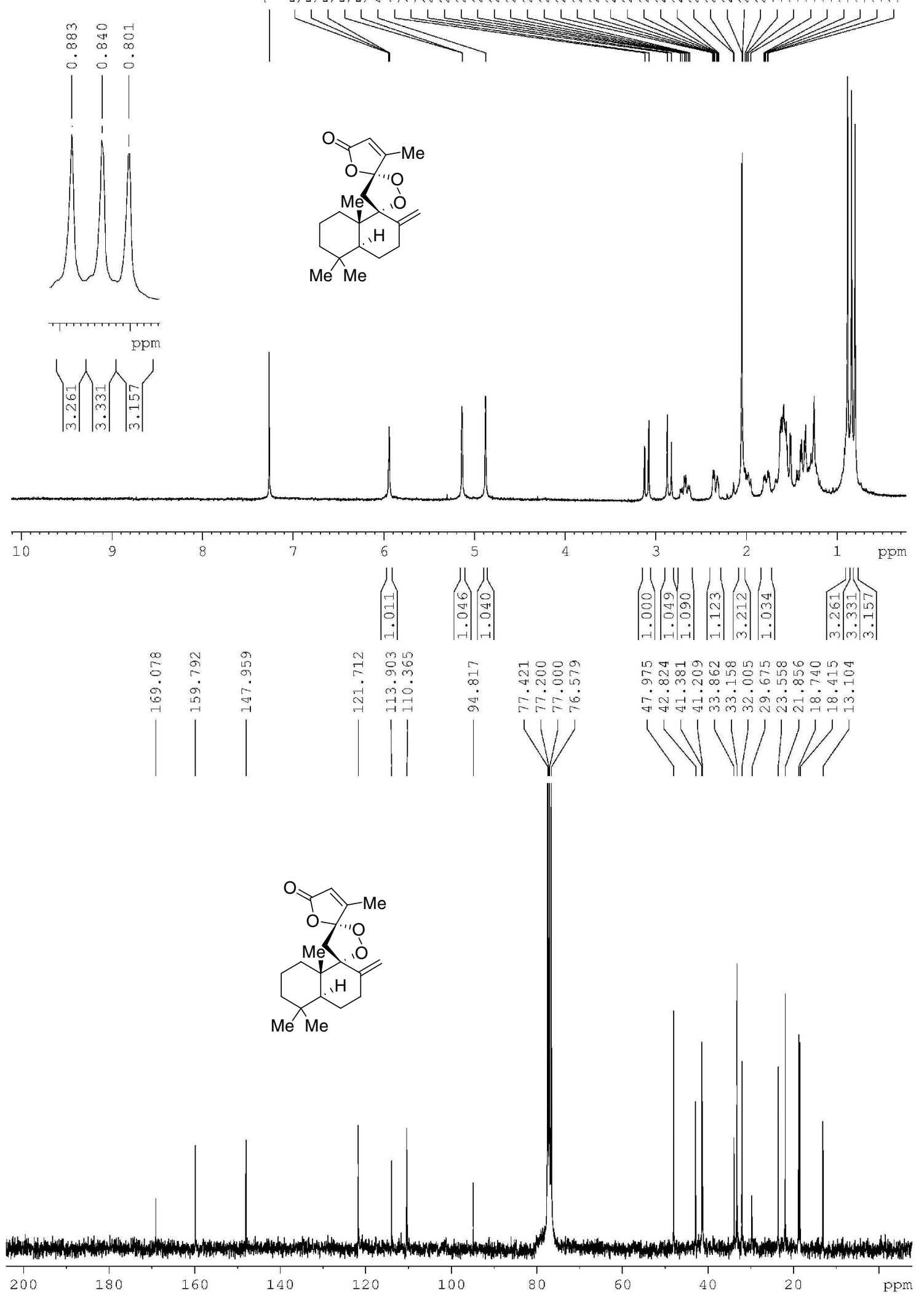



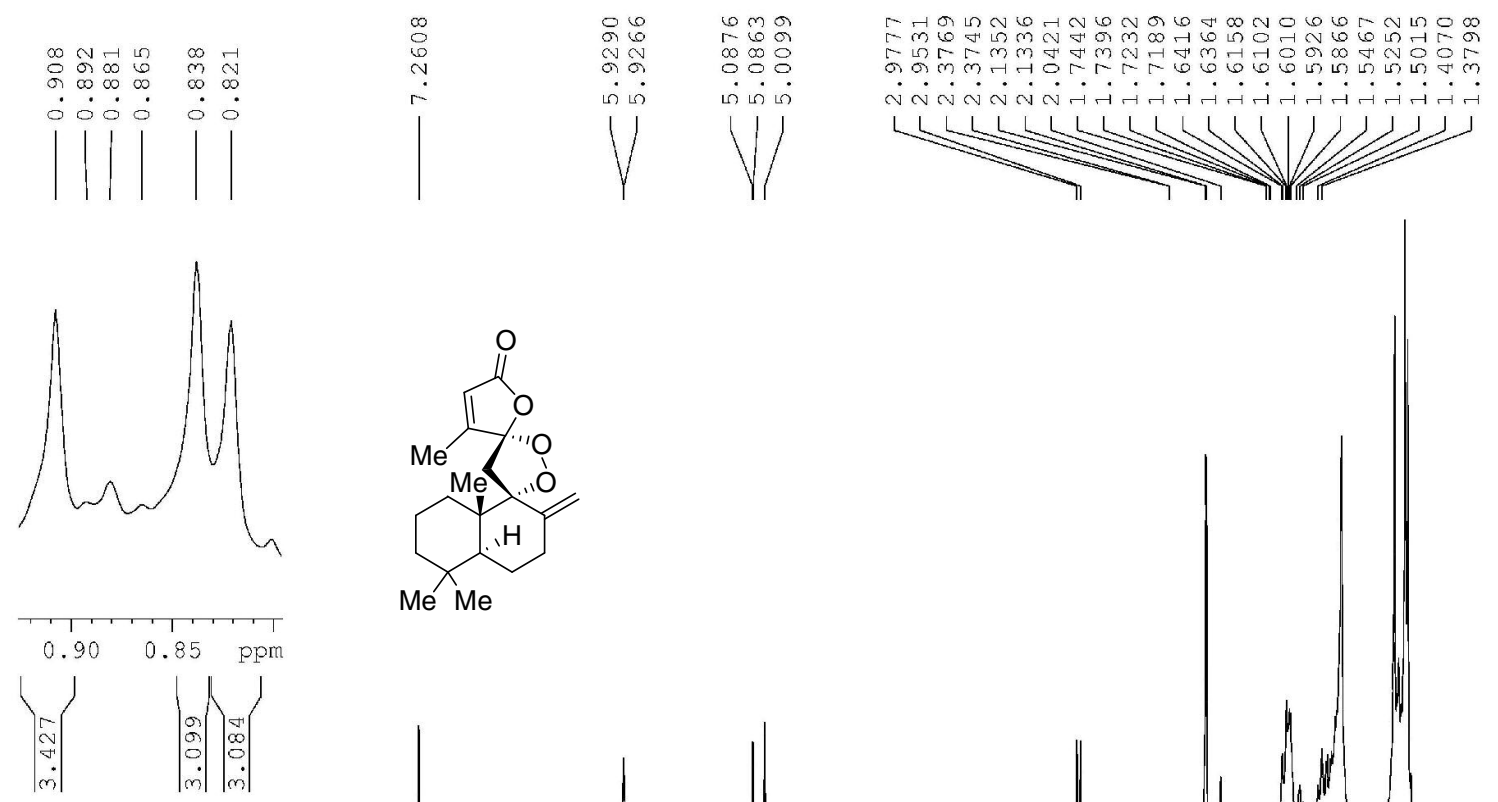